\title{
Kolmogorov complexity of enumerating finite sets
}

\author{
Nikolai K. Vereshchagin*
}

Keywords: Kolmogorov complexity, the a priori probability.

\begin{abstract}
Solovay [5] has proved that the minimal length of a program enumerating a set $A$ is upper bounded by 3 times the negative logarithm of the probability that a random program will enumerate $A$. It is unknown whether one can replace the constant 3 by a smaller constant. In this paper, we show that the constant 3 can be replaced by the constant 2 for finite sets $A$.
\end{abstract}

We recall first two complexity measures ("information content") of computably enumerable sets attributed by Solovay in [5] to G. Chaitin (wee keep Solovay's notations).

Let $M$ be a machine with one infinite input tape and one infinite output tape. At the start the input tape contains an infinite binary string $\omega$ called the input to $M$. The output tape is empty at the start. We say that a program $p$ enumerates a set $A \subset \mathbb{N}=\{1,2, \ldots\}$ if in the run on every input $\omega$ extending $p$ machine $M$ prints all the elements of $A$ in some order and no other elements, and does not move the head on input tape beyond $p$. We do not require $M$ to halt in the case when $A$ is finite. ${ }^{1}$ Let $I_{M}(A)$ denote the minimal length of a program enumerating $A$. There is a machine $M_{0}$ (called a universal machine) such that for every other machine $M$ there is a constant $c$ such that

$$
I_{M_{0}}(A) \leqslant I_{M}(A)+c
$$

for all $A \subset \mathbb{N}$. Fix any such $M_{0}$ and call $I(A) \stackrel{\text { def }}{=} I_{M_{0}}(A)$ the complexity of enumeration of $A$. This complexity thus depends on the choice of the universal machine but this dependence is rather weak: for any other universal machine $M_{1}$ the difference $\left|I_{M_{0}}(A)-I_{M_{1}}(A)\right|$ is bounded by a constant not depending on $A$.

The second complexity measure is related to the a priori probability distribution on enumerable sets. The definitions are as follows. Let $M$ be a machine

\footnotetext{
*Moscow State University, Leninskie Gory 1, Moscow 119992, Email: ver@mccme.ru. Work was done while visiting Laboratoire d'Informatique Fondamentale, Université de Provence. Supported in part by the RFBR grant 06-01-00122-a

${ }^{1}$ In the case of finite sets any such program is called an implicit description of $A$, as opposed to explicit description of $A$ when $M$ is required to halt after having printed the last element of $A$.
} 
with one infinite input tape and one infinite output tape as described above. For every infinite 0 -1-sequence $\omega$ let $M(\omega)$ denote the set enumerated by $M$ when $\omega$ is written on its input tape. For every $A \subset \mathbb{N}$ consider the probability

$$
m_{M}(A)=\operatorname{Pr}[M(\omega)=A] .
$$

A theorem of de Leeuw, Moore, Shannon and Shapiro [2] states that if $m_{M}(A)>$ 0 then $A$ is enumerable.

The class of distributions of such form has a maximal one up to a multiplicative constant. In other words, there is a machine $M_{1}$ (called optimal) such that for every machine $M$ there is a constant $c$ such that

$$
c \cdot m_{M_{1}}(A) \geqslant m_{M}(A)
$$

for all $A \subset \mathbb{N}$. Fix any such $M_{1}$ and call $m(A) \stackrel{\text { def }}{=} m_{M_{1}}(A)$ the a priori probability of enumerating $A$. The a priori distribution thus depends on the choice of the optimal machine but this dependence is also weak: for any other optimal machine $M_{2}$ both ratios $m_{M_{1}}(A) / m_{M_{2}}(A)$ and $m_{M_{2}}(A) / m_{M_{1}}(A)$ are bounded by a constant not depending on $A$. Let $H(A)$ denote the negative binary logarithm of the a-priori probability of $A$ : $H(A)=\lceil-\log m(A)\rceil$.

Comparing $M_{0}$, the machine defining $I(A)$, with $M_{1}$, the machine defining $m(A)$, we see that

$$
H(A)=\left\lceil-\log m_{M_{1}}(A)\right\rceil \leqslant I_{M_{1}}(A) \leqslant I_{M_{0}}(A)+O(1)=I(A)+O(1)
$$

for all $A$. Solovay [5] has proved that conversely $I(A) \leqslant 3 H(A)+O(\log H(A))$ for all $A$, which can be viewed as a sharpening of de Leeuw et al.'s result.

Theorem 1 (Solovay). There is a constant $c$ such that for every set $A \subset \mathbb{N}$ we have $I(A) \leqslant 3 H(A)+2 \log H(A)+c$.

It is unknown whether we can replace the constant 3 in this inequality by a smaller constant. In this paper, we show that the constant 3 can be replaced by the constant 2 for finite sets $A$.

Theorem 2. There is a constant $c$ such that for every finite set $A$ we have $I(A) \leqslant 2 H(A)+2 \log H(A)+c$.

The proof of Theorem 2 is basically a simplification of that of Theorem 1 . Thus we first sketch the latter one and then present the former one, explaining the main difference between two proofs. Our proof of Theorem 1 keeps the main ideas of Solovay's proof but differs from it in many technical details.

First we introduce some terminology and notation. Let $\Omega$ stand for the set of all infinite binary sequences. We write $x \leqslant \omega$ if $x$ is a finite prefix of an infinite 0-1-sequence $\omega$. Let $\Omega_{x}$ denote the set of all $\omega \in \Omega$ with $x \leqslant \omega$.

We will consider Cantor topology on $\Omega$. Its base open sets are all sets of the form $\Omega_{x}$. We call a subset of $\Omega$ finitely based if it is a finite union of sets of the form $\Omega_{x}$. 
There is a natural one-to one correspondence between the family of all subsets of $\mathbb{N}$ and $\Omega$. Each subset $A$ of $\mathbb{N}$ corresponds to its characteristic sequence, whose $n$th bit is 1 iff $n \in A$. In what follows we will identify subsets of $\Omega$ with its characteristic sequences. In particular, we will write $A \in \Omega_{x}$ and $x \leqslant A$ to indicate that $x$ is a prefix of the characteristic sequence of $A$. The notation $A \subset B$ will be used for the inclusion relation.

Fix and optimal machine $M$ defining the a priori distribution $m$. Let $M^{t}(\omega)$ stand for the set enumerated by $M$ in $t$ steps on input $\omega$. For each $A \subset \mathbb{N}$ and $t$ let

$$
S^{t}(A)=\left\{\omega \mid M^{t}(\omega)=A\right\} .
$$

The set $S^{t}(A)$ is finitely based and a code of $S^{t}(A)$ (a finite list of respective $x$ 's) can by computed given $t$ and $A$. (For all infinite $A$ and all $t$ the set $S^{t}(A)$ is empty.) Let $\mu$ denote the uniform measure on $\Omega$ and $m^{t}(A)=\mu\left(S^{t}(A)\right)$. According to our agreement to identify sets with their characteristic sequences, we denote by $m^{t}\left(\Omega_{x}\right)$ the total $m^{t}$-measure of all sets whose characteristic sequence begins with $x$.

Note that $m^{t}(A)$ can both increase and decrease as $t$ increases (if $A$ is finite). Indeed, assume that $M^{t-1}(\omega)=A$ and on step $t$ of the run on input $\omega$ the machine $M$ writes a new element $b$ on the output tape. Let $x$ be the length- $t$ prefix of $\omega$. Then $S(A)$ is decremented by $\Omega_{x}$, while $S(A \cup\{b\})$ is incremented by $\Omega_{x}$ on step $t$.

Proof of Theorem 1 (a sketch). Let $T$ be a subset of $\{0,1\}^{*}$. A limit point of $T$ is an infinite 0 -1-sequence $\omega$ having the following property: Every its prefix is a prefix of some string in $T$. By $T^{n}$ we denote the set of all strings of length $n$ in $T$. We say that an algorithm constructs a set $T$ of binary strings if for any given $n$ it prints the list of strings in $T^{n}$ and then halts.

An essential part of the proof is an algorithm that for every $k$ constructs a set of strings $T_{k}$ and computes a sequence of natural numbers

$$
t_{0}<t_{1}<t_{2}<\ldots
$$

having the following properties:

$$
\begin{aligned}
& m^{t_{n}}\left(\Omega_{x}\right) \geqslant 2^{-k-1} \text { for all } n \text { and all } x \in T_{k}^{n}, \\
& \text { if } m(A) \geqslant 2^{-k} \text { then } A \text { is a limit point of } T_{k} .
\end{aligned}
$$

Lemma 1. There is an algorithm that given $k$ and an auxiliary binary string $\lambda_{k}$ of length $k+1$ constructs a set $T_{k}$ and computes an increasing sequence $t_{0}, t_{1}, \ldots$ having the properties (1) and (2).

For the goal of this paper, we do not need the proof of this lemma. However, for the seek of completeness we present its proof in the Appendix.

So, assume Lemma 1. We are going to construct an algorithm that on input $\lambda_{k}$ enumerates certain subsets $C_{1}, \ldots, C_{N}$ of $\mathbb{N}$, where $N=O\left(2^{2 k}\right)$, having the following property: Every limit point $A$ of $T_{k}$ (the tree constructed by the algorithm of Lemma 1) is among $C_{1}, \ldots, C_{N}$. 
To this end we need a computable strategy to win an infinite two person game defined in Martin's paper [4]. Let $N, K$ be natural numbers. A configuration in this game is an $N$-tuple of finitely based subsets of $\Omega$ : $\left\langle Z_{1}, \ldots, Z_{N}\right\rangle$. The initial configuration is $\langle\Omega, \ldots, \Omega\rangle$. Player I on his turn plays a finitely based set $Y$ with $\mu(Y) \geqslant 1 / K$. If $Y$ is disjoint with $Z_{i}$ for all $i=1, \ldots, N$, player I wins. If not, player II chooses a $Z_{i}$ intersecting $Y$ and replaces $Z_{i}$ by $Y$, and the game continues. Player II wins if he can prevent I to win as described above for the entire, infinitely long game.

Martin [4] has proved that if $N=K(K+1) / 2$ then player II has a computable winning strategy (uniformly in $K$ ). We use Martin's result for $K=$ $2^{k+1}$. (We present its proof in the Appendix.)

Algorithm. We make steps $n=1,2, \ldots$ At the end of step $n$ we will have a configuration $\left\langle Z_{1}, \ldots, Z_{N}\right\rangle$ in Martin's game and the sets $C_{1}, \ldots, C_{N}$ enumerated so far. They will satisfy the following conditions:

$C_{i} \subset\{1, \ldots, n\}$ for all $i \leqslant N$;

$C_{i} \subset M^{t_{n}}(\omega)$ for all $\omega \in Z_{i}$ and all $i \leqslant N$;

every $x \in T_{k}^{n}$ is a prefix of $C_{i}$ for some $i \leqslant N$;

the configuration $\left\langle Z_{1}, \ldots, Z_{N}\right\rangle$ was obtained by applying a computable

winning strategy of player II against a sequence of moves of player I.

At the start $n=0, C_{i}=\emptyset$ and $Z_{i}=\Omega$, and conditions (3), (4), (5) and (6) are straightforward.

Step $n$. At the beginning of step $n$ the conditions (3), (4), (5) and (6) are true for $n-1$. The conditions (3) and (4) for $n-1$ imply conditions (3) and (4) for $n$. Thus all the conditions except (5) are true at the beginning of step $n$. The condition (5) however may become false for any of $x \in T_{k}^{n}$, as $T_{k}^{n}$ and $T_{k}^{n-1}$ can be unrelated.

How to restore condition (5)? First, using the algorithm of Lemma 1, find the list of $T_{k}^{n}$. Then pick any $x$ from $T_{k}^{n}$, call it $x_{1}$. Play $Y_{1}=S^{t_{n}}\left(\Omega_{x_{1}}\right)$ for player I in Martin's game. By condition (1) this is a legal move. Assume that the winning strategy of player II plays $Z_{i}$.

We want to add some elements to $C_{i}$ to ensure $x_{1} \leqslant C_{i}$. To this end we need to show that $C_{i}$ is a subset of the set $X_{1}=\left\{j \mid j\right.$ th bit of $x_{1}$ is 1$\}$. We are given that there is $\omega \in Z_{i}$ which is in $Y_{1}$, that is,

$$
x_{1} \leqslant M^{t_{n}}(\omega) \text {. }
$$

By condition (4) we have

$$
C_{i} \subset M^{t_{n}}(\omega) .
$$

Thus $C_{i}$ is a subset of a set whose charactersitic sequence begins with $x_{1}$, therefore $C_{i} \cap\{1, \ldots, n\} \subset X_{1}$. By conditon (3) this implies that thus $C_{i} \subset X_{1}$.

Update $C_{i}$ by letting $C_{i}=X_{1}$. Thus condition (5) is fulfilled for $x=x_{1}$.

Replace $Z_{i}$ by $Y_{1}$. This replacement restores conditon (4) (that might become false after changing $\left.C_{i}\right)$. Indeed, $x_{1} \leqslant M^{t_{n}}(\omega)$ for all $\omega \in Y_{1}$ and $C_{i}$ has just become equal to $X_{1}$. 
Next we pick another element $x_{2}$ from $T_{k}^{n}$ and repeat the procedure for $x_{2}$ in place of $x_{1}$. Note that the set $Y_{2}$ is disjoint with $Y_{1}$, as $M^{t_{n}}\left(Y_{2}\right)$ and $M^{t_{n}}\left(Y_{1}\right)$ are equal to disjoint sets $\Omega_{x_{2}}$ and $\Omega_{x_{1}}$. Thus the new move $Z_{i}$ of player II is different from $Y_{1}$ and the condition (5) remains true for $x=x_{1}$. Repeating the procedure $\left|T_{k}^{n}\right|$ times we fulfil condition (5) for all $x \in T_{k}^{n}$. End of Algorithm.

We need to prove that for every limit point $A$ of $T_{k}$ there is $i$ with $C_{i}=A$. For every prefix $x$ of (the characteristic sequence of) $A$, at the end of some step $n \geqslant|x|$ the string $x$ is a prefix of $C_{i}$ for some $i$. That $i$ may depend on $x$. However, as the number of possible $i$ 's is finite, there is $i$ such that every prefix $x$ of $A$ is a prefix of $C_{i}$ at the end of some step $n \geqslant|x|$.

This obviously implies that $A \subset C_{i}$. To prove the converse inclusion, pick any $j \in C_{i}$ and assume that $j$ was included in $C_{i}$ on the step $m$ (thus $m \geqslant j$ ). The length- $m$ prefix of $A$ is a prefix of $C_{i}$ at the end of some step $n \geqslant m$. Thus $j$ th bit of the characteristic function of $A$ is 1 .

Consider the machine that on every input $\omega$ beginning with

$$
\left.p=0^{\log k} 1 \text { (binary notation of } k\right)\left(\lambda_{k}\right) \text { (binary notation of } i \text { ) }
$$

scans $p$ and then, running the Algorithm, enumerates the set $C_{i}$ (and no other sets among $\left.C_{1}, \ldots, C_{N}\right)$. For this machine $M^{\prime}$ it holds

$$
I_{M^{\prime}}\left(C_{i}\right) \leqslant 2 \log k+1+k+1+\log O\left(2^{2 k}\right)
$$

and by universality

$$
I\left(C_{i}\right) \leqslant I_{M^{\prime}}\left(C_{i}\right)+O(1) \leqslant 3 k+2 \log k+O(1)
$$

for all $i$.

Let $A$ be any enumerable subset of $\mathbb{N}$ and $k=H(A)$. By condition (2) there is $i$ such that the set $C_{i}$ enumerated by the Algorithm coincides with $A$. Thus we obtain

$$
I(A) \leqslant 3 H(A)+2 \log H(A)+O(1) .
$$

How can we improve Solovay's bound

$$
I(A) \leqslant 3 H(A)+2 \log H(A)+O(1) ?
$$

We could try to improve the upper bound of $N$ in Martin's game. However, Ageev [1] showed that the condition $N=\Omega\left(K^{2}\right)$ is necessary for player II to win. Another option would be to reduce the length of the auxiliary string $\lambda_{k}$ in Lemma 1. We do not know if this is possible.

For finite sets we can simplify the above construction as follows. If $A$ is finite and $m(A) \geqslant 2^{-k}$ then $m^{t}(A) \geqslant 2^{-k-1}$ for all large enough $t$ (Lemma 2 below). Thus we do not need the algorithm of Lemma 1 . The algorithm that enumerates sets $C_{1}, \ldots, C_{N}$ enforces, on step $t$, that every finite $A$ with $m^{t}(A) \geqslant 2^{-k-1}$ be among $C_{1}, \ldots, C_{N}$. Thus we get rid of the string $\lambda_{k}$, and the enumeration complexity of $C_{1}, \ldots, C_{N}$ is reduced by about $k$ bits. 
Proof of Theorem 2. We construct an algorithm that given $k$ enumerates $N=O\left(2^{2 k}\right)$ sets $C_{1}, \ldots, C_{N}$ so that every finite set $A$ with $m(A) \geqslant 2^{-k}$ coincides with $C_{i}$ for some $i \leqslant N$. Just as in the proof of Theorem 1 this implies

$$
I(A) \leqslant 2 H(A)+2 \log H(A)+O(1) .
$$

Algorithm. We make steps $t=1,2, \ldots$ At the end of each step $t$ we will have a configuration $\left\langle Z_{1}, \ldots, Z_{N}\right\rangle$ in Martin's game for $K=2^{k+1}$ and the sets $C_{1}, \ldots, C_{N}$ enumerated so far. They will satisfy the following conditions:

$C_{i} \subset M^{t}(\omega)$ for all $\omega \in Z_{i}$ and all $i=1, \ldots, N$,

every finite $A$ with $m^{t}(A) \geqslant 2^{-k-1}$ is among $C_{1}, \ldots, C_{N}$,

the configuration $\left\langle Z_{1}, \ldots, Z_{N}\right\rangle$ was obtained by applying a computable winning strategy of player II against a sequence of moves of player I.

Step $t$. At the beginning of step $t$ conditions (7), (8) and (9) are true for $t-1$. Obviously, condition (7) remains valid for $t$ in place of $t-1$, and we need to restore condition (8).

First we find all sets $A_{1}, \ldots, A_{s}$ with

$$
m^{t}\left(A_{i}\right) \geqslant 2^{-k-1} .
$$

Then we play $Y_{1}=S^{t}\left(A_{1}\right)$ for the player I in Martin's game. Let $Z_{i}$ be the move of the computable winning strategy of player II. As $Z_{i}$ intersects $Y$, there is $\omega \in Z_{i}$ with $A_{1}=M^{t}(\omega)$. By condition (7) we have

$$
C_{i} \subset M^{t}(\omega)=A_{1} .
$$

We update $C_{i}$ be letting $C_{i}=A_{1}$ and replace $Z_{i}$ by $Y_{1}$. These changes enforce condition (8) for $A=A_{1}$ and do not break condition (7).

Then we repeat the procedure for $A_{2}, \ldots, A_{s}$ in place of $A_{1}$. Note that $S^{t}\left(A_{1}\right), \ldots, S^{t}\left(A_{s}\right)$ are pairwise disjoint. Therefore, the condition (8) for $A_{1}, \ldots, A_{i-1}$ will not be broken when we perform the procedure for $A_{i}$. End of Algorithm.

The correctness of the Algorithm is based on the following

Lemma 2. $m(A)=\lim _{t \rightarrow \infty} m^{t}(A)$ for every finite $A \subset \mathbb{N}$.

The proof of this lemma is an easy exercise in measure theory and is given in the Appendix.

If $A$ is finite and $m(A) \geqslant 2^{-k}$ then by Lemma 2 for almost all $t$ we have $m^{t}(A) \geqslant 2^{-k-1}$. Therefore there is $i$ such that on infinitely many steps we have $C_{i}=A$. Since $C_{i}$ can only increase on each step, $A$ coincides with $C_{i}$ starting from some step.

Acknowledgments. The author is sincerely grateful to Sergei Salnikov for writing down a preliminary version of the proof and for the anonymous referees for helpful suggestions. 


\section{References}

[1] M. Ageev, Martin's game: a lower bound for the number of sets, Theor. Comput. Sci. 289(1): 871-876 (2002)

[2] K. de Leeuw, E. F. Moore, C. E. Shannon, and N. Shapiro, Computability by probabilistic machines, In: C. E. Shannon and J. McCarthey (Eds.), Automata Studies, Princeton University Press, Princeton, New Jersey, 1956, $183-212$.

[3] M. Li and P.M.B. Vitányi, An Introduction to Kolmogorov Complexity and its Applications, Springer-Verlag, New York, 2nd Edition, 1997.

[4] D.A. Martin, Borel indeterminacy, Ann. Math. 102 (1978) 363-371.

[5] R.M.Solovay, On Random R.E. Sets, In: A.I. Arruda, N.C.A. da Costa, R. Chaqui (Eds.), Non-Classical Logics, Model Theory and Computability, North-Holland, Amsterdam, 1977, pp. 283-307.

\section{Appendix.}

Proof of Lemma 2. The set $S^{t}(A)$ is the difference of two sets: $S_{1}^{t}=\{\omega \mid M(\omega)$ prints in at most $t$ steps all the elements of $A\}$ and $S_{2}^{t}=\left\{\omega \mid M_{1}(\omega)\right.$ prints in at most $t$ steps all the elements of $A$ and an element of $\mathbb{N} \backslash A\}$. Let $S_{1}^{\infty}$ be the union of all $S_{1}^{t}$ and $S_{2}^{\infty}$ the union of all $S_{2}^{t}$. As the uniform measure is continuous we have

$$
\mu\left(S_{1}^{\infty}\right)=\lim _{t \rightarrow \infty} \mu\left(S_{1}^{t}\right), \quad \mu\left(S_{2}^{\infty}\right)=\lim _{t \rightarrow \infty} \mu\left(S_{2}^{t}\right),
$$

and

$$
\begin{aligned}
m(A) & =\mu\left(S_{1}^{\infty} \backslash S_{2}^{\infty}\right) \\
& =\mu\left(S_{1}^{\infty}\right)-\mu\left(S_{2}^{\infty}\right) \\
& =\lim _{t \rightarrow \infty} \mu\left(S_{1}^{t}\right)-\lim _{t \rightarrow \infty} \mu\left(S_{2}^{t}\right) \\
& =\lim _{t \rightarrow \infty}\left(\mu\left(S_{1}^{t}\right)-\mu\left(S_{2}^{t}\right)\right) \\
& =\lim _{t \rightarrow \infty} \mu\left(S_{1}^{t} \backslash S_{2}^{t}\right)=\lim _{t \rightarrow \infty} \mu\left(S^{t}(A)\right)=\lim _{t \rightarrow \infty} m^{t}(A) .
\end{aligned}
$$

Proof of Lemma 1. Let $A_{1}, \ldots, A_{r}$ be all sets with $m\left(A_{i}\right) \geqslant 2^{-k}$ and let $\lambda=$ $\sum_{i=1}^{r} m\left(A_{i}\right)$. Let $\lambda_{k}$ be the rational number consisting of $k+1$ first binary digits of the number $\lambda-2^{-k-1}$.

The number $t_{n}$ and the list $T_{k}^{n}$ of all strings of length $n$ in $T_{k}$ are defined recursively. Let $t_{n}$ be the first $t>t_{n-1}$ such that

$$
m^{t}\left(\Omega_{x_{1}} \cup \cdots \cup \Omega_{x_{s}}\right) \geqslant \lambda_{k},
$$


where $x_{1}, \ldots, x_{s}$ are all binary strings of length $n$ with

$$
m^{t}\left(\Omega_{x_{i}}\right) \geqslant \max \left\{2^{-k-1}, 2^{-k}-1 / n\right\} .
$$

Let $T_{k}^{n}=\left\{x_{1}, \ldots, x_{s}\right\}$.

We have to prove first that such $t$ exists. Let $x_{1}^{n}, \ldots, x_{r}^{n}$ stand for prefixes of length $n$ of characteristic functions of $A_{1}, \ldots, A_{r}$ (some of them may coincide). For any finitely based set $S \subset \Omega$ we have

$$
m(S)=\lim _{t \rightarrow \infty} m^{t}(S) .
$$

(This can be proved just as Lemma 2.) This implies that for all large enough $t$ we have

$$
m^{t}\left(\Omega_{x_{1}^{n}} \cup \cdots \cup \Omega_{x_{r}^{n}}\right) \geqslant \lambda_{k}
$$

We can pick $t$ so large that we additionally have

$$
m^{t}\left(\Omega_{x_{i}^{n}}\right) \geqslant \max \left\{2^{-k-1}, 2^{-k}-1 / n\right\} .
$$

for all $i=1, \ldots, r$. Indeed, $m^{t}\left(\Omega_{x_{i}^{n}}\right)$ tends to $m\left(\Omega_{x_{i}^{n}}\right)$, which is at least $m\left(\Omega_{A_{i}}\right) \geqslant 2^{-k}$. Any such $t$ qualifies all the requirements.

It remains to prove that every $A$ with $m(A) \geqslant 2^{-k}$ is a limit point of $T_{k}$. Let $B_{1}, \ldots, B_{m}$ be all different limit points of $T_{k}$. It suffices to show that

$$
m\left(\left\{B_{1}, \ldots, B_{m}\right\}\right) \geqslant \lambda_{k} \text { and } m\left(B_{i}\right) \geqslant 2^{-k}
$$

for all $i \leqslant m$. (Indeed, if $A$ was not among $B_{1}, \ldots, B_{m}$, then the $m$-measure of the set of all $B$ 's with $m(B) \geqslant 2^{-k}$ would be at least $\lambda_{k}+2^{-k}>\lambda$.)

Let us prove first that $m\left(B_{i}\right) \geqslant 2^{-k}$ for every $i \leqslant m$. Fix $i$ and let $z_{i}^{n}$ denote length- $n$ prefix of $B_{i}$. As $m$ is a continuous measure, we have

$$
m\left(B_{i}\right)=\lim _{n \rightarrow \infty} m\left(\Omega_{z_{i}^{n}}\right) .
$$

Thus it suffices to prove that $m\left(\Omega_{z_{i}^{n}}\right) \geqslant 2^{-k}$ for all $n$. Fix $n$. As $B_{i}$ is a limit point of $T_{k}$, for every $j \geqslant n$ there exists $l \geqslant j$ such that $z_{i}^{j}$ is a prefix of some $x \in T_{k}^{l}$. This implies that

$$
m^{t_{l}}\left(\Omega_{z_{i}^{n}}\right) \geqslant m^{t_{l}}\left(\Omega_{z_{i}^{j}}\right) \geqslant m^{t_{l}}\left(\Omega_{x}\right) \geqslant 2^{-k}-1 / l .
$$

Thus for every $j \geqslant n$ there is $l \geqslant j$ with

$$
m^{t_{l}}\left(\Omega_{z_{i}^{n}}\right) \geqslant 2^{-k}-1 / l,
$$

which implies that $m\left(\Omega_{z_{i}^{n}}\right) \geqslant 2^{-k}$.

The inequality $m\left(\left\{B_{1}, \ldots, B_{m}\right\}\right) \geqslant \lambda_{k}$ is proved in a similar way. Let $S_{n}$ stand for the set of all $\omega$ whose length- $n$ prefix is among of $z_{1}^{n}, \ldots, z_{m}^{n}$ :

$$
S_{n}=\Omega_{z_{1}^{n}} \cup \cdots \cup \Omega_{z_{m}^{n}} .
$$


The $m$-measure of $S_{n}$ tends to $m\left(\left\{B_{1}, \ldots, B_{m}\right\}\right)$, thus it suffices to show that

$$
m\left(S_{n}\right) \geqslant \lambda_{k}
$$

for all $n$.

Fix any $n$. For all large enough $l$ every string $x$ of length $l$ in $T_{k}$ is an extension of some string among $z_{1}^{n}, \ldots, z_{m}^{n}$. (Otherwise, compactness arguments show that $T_{k}$ has a limit point outside $S_{n}$, hence different from $\left.B_{1}, \ldots, B_{m}\right)$. Fix any such $l$ and let $x_{1}, \ldots, x_{s}$ denote all strings of length $l$ in $T_{k}$. By construction we have

$$
m^{t_{l}}\left(\Omega_{x_{1}} \cup \cdots \cup \Omega_{x_{s}}\right) \geqslant \lambda_{k}
$$

and therefore

$$
m^{t_{l}}\left(S_{n}\right) \geqslant m^{t_{l}}\left(\Omega_{x_{1}} \cup \cdots \cup \Omega_{x_{s}}\right) \geqslant \lambda_{k} .
$$

Since this inequality holds for all large enough $l$ and $m\left(S_{n}\right)=\lim _{t \rightarrow \infty} m^{t}\left(S_{n}\right)$, we are done.

How to win Martin's game? Without loss of generality we may assume that the measure of every move $Y$ of player $\mathrm{I}$ is exactly $1 / K$ and thus at any moment of the game the measure of all sets $Z_{1}, \ldots, Z_{N}$ is exactly $1 / K$. (At the start of the game we will reduce $Z_{1}, \ldots, Z_{N}$; if we win the game with reduced $Z_{1}, \ldots, Z_{N}$ then we certainly win with original $Z_{1}, \ldots, Z_{N}$.)

We assign to every set $Z_{i}$ a natural number in the range $1, \ldots, K$, called the rank, so that (1) for every $r \leqslant K$ there are exaclty $r$ sets of rank $r$ and (2) all sets of the same rank are pair wise disjoint (thus sets of rank $K$ form a partition of $\Omega$ ).

The condition (2) implies that each move $Y$ of player II intersects some set of rank $K$. On our next move we choose a set $Z_{i}$ of lowest rank $r$ that intersects $Y$, replace it by $Y$ and assign the rank $r-1$ to $Y$. The condition (2) is thus satisfied. Note that now there are $r$ (pair wise disjoint sets) of rank $r-1$ and $r-1$ sets of rank $r$. Thus swapping sets of rank $r$ and $r-1$ restores the condition (1). 